\title{
Factores de fatiga en operadores de maquinaria semiautomatizada en México
}

\author{
Juan Luis Hernández Arellano \\ Universidad Autónoma de Ciudad Juárez. Chihuahua, México. \\ Correo electrónico: luis.hernandez@uacj.mx \\ María Julia Brunette \\ Universidad de Massachusetts Lowell. Massachusetts, Estados Unidos \\ Correo electrónico: maria_brunette@uml.edu \\ Gabriel Ibarra Mejía \\ Universidad Autónoma de Ciudad Juárez. Chihuahua, México \\ Correo electrónico: gabriel.ibarra@uacj.mx \\ Jorge Luis García Alcaraz \\ Universidad Autónoma de Ciudad Juárez. Chihuahua, México \\ Correo electrónico: jorge.garcia@uacj.mx.
}

Recibido: 24/4/2012 / Aprobado: 12/6/2012

Resumen: En este estudio se aplicó la versión en castellano del Inventario Sueco de Fatiga Ocupacional (Swedish Occupational Fatigue InventorySpanish), con el objetivo de identificar los síntomas de fatiga en operadores de maquinaria semiautomatizada clasificada como tecnología de manufactura avanzada (TMA). Se encuestaron 121 operadores de tornos de control numérico computarizado (CNC) en una empresa fabricante de flechas homocinéticas localizada en la ciudad de Celaya, Guanajuato, en el centro de México. Los resultados muestran que el factor falta de energía se relaciona con un nivel más alto de fatiga, mientras que el factor somnolencia se relacionó con un nivel más bajo de fatiga.

Palabras clave: fatiga mental / esfuerzo físico / TMA / cuestionario SOFI-S

\section{Fatigue factors in semiautomated machine operators in Mexico}

AвsTRACT: In this study the Swedish Occupational Fatigue InventorySpanish version (SOFI-S) was applied in order to identify the symptoms of fatigue between Advances Manufacturing Technology (AMT) operators in Mexico. A total of 121 Computerized Numeric Control (CNC) lathe operators were surveyed in a manufacturer of Constant Velocity (CV) joints located in Celaya, Guanajuato in central Mexico. Typical tasks performed by operators included loading, unloading and machine programming. Results show that the dimension "lack of energy" obtained the highest score of fatigue, while the "lack of motivation" obtained the lowest score of fatigue.

Keywords: mental fatigue / physical effort / TMA / SOFI-S questionnaire 


\section{INTRODUCCIÓN}

Con la introducción de nuevas y cada vez más sofisticadas tecnologías, realizar el trabajo con máquinas consideradas como TMA es una tarea cada vez más compleja (DiDomenico \& Nusbaum, 2008), sobre todo en actividades que demandan la realización de esfuerzos físicos y mentales de forma combinada. Un ejemplo de este tipo de trabajos es la operación de tornos de CNC, que son máquinas clasificadas como TMA. Al respecto, Farrer (1994), Mondelo (1996), Mital (1995) y Mital \& Pennathur (2004) postulan que en los trabajos en que se realizan esfuerzos físicos y mentales la aparición de fatiga puede ocurrir prematuramente.

El interés por investigar la valoración de fatiga se ha incrementado en los últimos años, sobre todo con la llegada de la tecnología a los ambientes industriales (DiDomenico \& Nusbaum, 2011). Mital (1995) concluye que la relación hombre-máquina, cuando esta es clasificada como TMA, debe ser investigada científicamente. De una serie de líneas de investigación que propuso como resultado de un taller de investigación sobre la interacción hombre-máquina-TMA, las siguientes tres preguntas son de particular interés en este estudio:

- ¿Cómo medir/valorar la fatiga física y mental en los trabajadores?

- ¿Cómo balancear la carga física y mental de trabajo?

- ¿Cuáles son los efectos del uso de TMA en la fatiga y desempeño de los trabajadores?

La investigación de fatiga en trabajos/actividades donde se realizan esfuerzos físicos y mentales, y además se incluye TMA, es escasa, especialmente en países en vías de desarrollo (Gónzalez \& Gutiérrez, 2006). Para el caso de México se han identificado pocas investigaciones que aborden estas condiciones de trabajo. Algunas de las publicaciones más relevantes son la validación del punto de corte de la Prueba de Síntomas Subjetivos de Fatiga (PSSF) en una muestra de trabajadores mexicanos (Barrientos, 2004), la influencia de la carga mental de trabajo como factor de riesgo de estrés (González \& Gutiérrez, 2004), la validación de la Escala de Puntos Estimados de Fatiga-Energía (ESPEFE); (Juárez-García, 2007) y la determinación de curvas de fatiga en mujeres empacadoras de tomate en el estado de Jalisco, México (Hernández et al., 2009).

La valoración de la fatiga se ha desarrollado principalmente utilizando cuestionarios validados estadísticamente. Uno de los primeros 
cuestionarios desarrollados es la Prueba de Síntomas Subjetivos de Fatiga (PSSF) (Yoshitake, 1978), que permite valorar la fatiga en los factores somnolencia y apatía, incapacidad para concentrarse y discomfort físico, y que es valorado de forma dicotómica. El método de evaluación ergonómica desarrollado por el Laboratorio de Economía y Sociología del Trabajo de Francia (LEST) (Guelaud, 1977) incluye los factores carga física, carga mental, ambiente físico, aspectos psicosociales y tiempo de trabajo y es medido de forma cualitativa y cuantitativa. Por otro lado, el método Cooper Harper (Wiewille \& Casali, 1983) es una escala de 10 divisiones con una expresión lingüística para cada una de estas. El SOFI (Ahsberg, 1997) es un cuestionario que incluye cinco factores falta de energía, esfuerzo físico, discomfort físico, falta de motivación y somnolencia, a su vez, cada factor incluye 3 ítems. La Escala de Valoración de Fatiga (Fatigue Assessment Scale, FAS) (Michielsen, 2003) fue desarrollado con el objetivo de obtener una valoración de fatiga total e incluye los factores fatiga mental y fatiga física. La versión en castellano del cuestionario SOFI, el SOFI-Spanish (SOFI-S) (González, 2005) incluye los mismos factores e ítems que la versión del año 2000 de Ahsberg et al. La Escala de Recuperación por Fatiga Ocupacional (Occupational Fatigue Exhaustion Recovery Scale, OFERS) (Winwood et al., 2005) incluye los factores fatiga aguda, fatiga crónica y cambio de turnos y es valorado por una escala Likert. Por último, la Escala Subjetiva de Puntos Estimados de Fatiga-Energía (ESPEFE) (Juárez-García, 2007) es un método que utiliza como base la escala de percepción de esfuerzo de Borg (Borg, 1990) y relaciona la percepción de fatiga con la energía que dispone un trabajador para realizar sus actividades. El último método mencionado es el único que ha sido identificado para la valoración de fatiga que se ha desarrollado para la población de México, debido al poco desarrollo de instrumentos en castellano, sobre todo en América Latina (Gonzalez et al, 2005; González \& Gutiérrez, 2006).

A pesar de contar con varios métodos desarrollados para la valoración de fatiga, el cuestionario SOFI es uno de los métodos más confiables (Leung, 2004; González, 2005) y ha tenido aplicaciones en diferentes ambientes (Ahsberg, 1997; Ahsberg, 2000; Leung, 2004; González, 2005; Barker, 2011; Hernández, 2012). La investigación que dio origen al cuestionario incluyó trabajos y actividades muy diversas, como las que realizan profesores, choferes de autobús, bomberos, trabajos al aire libre, enfermeras, operadores de plantas nucleares, entre otros 
(Ahsberg, 1997). Todas las actividades que desarrollaron los trabajadores tuvieron como característica principal ser labores con cargas de trabajo (física o mental) significativas. La revisión de este instrumento en el año 2000, por Ahsberg, incluyó las actividades desarrolladas por profesores, bomberos, cajeros de supermercados, choferes de autobús e ingenieros de producción. En esta nueva versión se redujeron de 5 a 4 los ítems incluidos en cada factor de fatiga. El cuestionario SOFI se ha utilizado para la valoración de fatiga en ambientes de la salud, tales como con pacientes con cáncer (Ahsberg \& Fürst, 2001; Fürst \& Ahsberg, 2001; Servaes et al., 2001 y Roscoe et al., 2007), con enfermeras (Barker, 2011; Gonzalez, 2005) y en experimentos de laboratorio (Barker, 2011), además de la validación para trabajadores mexicanos operadores de tornos CNC (Hernández et al., 2012). Las 2 traducciones a idiomas diferentes al inglés que se han identificado del cuestionario SOFI han sido la versión para China (Leung, 2004) y la castellana (González, 2005). Para la versión en el idioma chino se encuestaron usuarios de equipos de cómputo y la versión en castellano fue desarrollada mediante la encuesta a enfermeras.

Esta investigación describe la aplicación del cuestionario SOFI-S en la operación de maquinaría clasificada como TMA en México (tornos $\mathrm{CNC}$ ). El objetivo principal es determinar los síntomas de fatiga (factores e ítems) entre operadores de tornos CNC que trabajan bajo el esquema de turnos extendidos de doce horas.

\section{METODOLOGÍA}

\subsection{Diseño del estudio}

La encuesta se aplicó entre operadores de tornos CNC y solamente se reportan aquí los resultados descriptivos y las correlaciones entre las variables y las dimensiones de fatiga analizadas. Se presenta aquí un estudio transversal, descriptivo y correlacional.

\subsection{Muestra}

Se encuestaron operadores de tornos CNC en 2 líneas de producción que realizan el maquinado de piezas llamadas "campana" y "tulipán". Los criterios de inclusión para que los trabajadores participaran en el estudio fueron los siguientes:

- Tener como mínimo 6 meses de laborar en la empresa como opera- 
dor de tornos CNC.

- Contar con carrera técnica/bachillerato en máquinas y herramientas.

- Contar con capacitación para ser operador de tornos CNC dentro de la empresa.

- Realizar actividades de carga, descarga y programación de tornos CNC.

La empresa cuenta con un total de 387 trabajadores operativos, de los cuales 287 (74\%) son operadores de tornos CNC, 85 (22\%) son operadores de robots totalmente automatizados, y 15 (4\%) son supervisores de líneas de producción. En esta investigación son de particular interés los operadores de tornos $\mathrm{CNC}$, debido a que realizan esfuerzos físicos y mentales de forma combinada.

\subsection{Valoración de fatiga}

Para la valoración de fatiga se utilizó la versión en español del cuestionario SOFI-S (González, 2005) validada para trabajadores mexicanos por Hernández (2012). Esta versión considera cinco factores: falta de energía, esfuerzo físico, discomfort físico, falta de motivación y somnolencia. Los ítems que se incluyen en cada una de las dimensiones se muestran en el cuadro 1.

\section{Cuadro 1}

Factores e ítems analizados en el estudio

\begin{tabular}{|l|l|}
\hline Factores & Ítems \\
\hline Falta de energía & $\begin{array}{l}\text { Agotado } \\
\text { Exhausto } \\
\text { Extenuado }\end{array}$ \\
\hline Esfuerzo físico & $\begin{array}{l}\text { Respirando con dificultad } \\
\text { Con palpitaciones } \\
\text { Con calor }\end{array}$ \\
\hline Discomfort físico & $\begin{array}{l}\text { Con las articulaciones engarrotadas } \\
\text { Entumecido } \\
\text { Adolorido }\end{array}$ \\
\hline
\end{tabular}




\begin{tabular}{|l|l|}
\hline Falta de motivación & $\begin{array}{l}\text { Pasivo } \\
\text { Apático } \\
\text { Indiferente }\end{array}$ \\
\hline Somnolencia & $\begin{array}{l}\text { Somnoliento } \\
\text { Durmiéndose } \\
\text { Bostezante }\end{array}$ \\
\hline
\end{tabular}

Fuente: Hernández et al., 2012.

Para la valoración de los ítems incluidos, se utilizó una escala de 5 puntos de respuesta que incluye las expresiones "bajo" en la parte izquierda y "alto" en la parte derecha. Se les pidió a los trabajadores que contestaran la encuesta de acuerdo a su percepción de fatiga al final de la jornada de trabajo de 12 horas.

\subsection{Aplicación de la encuesta}

El proyecto estuvo respaldado por la gerencia de una empresa fabricante de flechas homocinéticas ubicada en la ciudad de Celaya, Guanajuato, en el centro de México. La encuesta fue entregada al equipo de supervisores de producción y ellos fueron los responsables de distribuir la encuesta a los trabajadores que participaron. Previo a la distribución, y con el objetivo de minimizar las dudas sobre las preguntas incluidas en el cuestionario, los trabajadores y supervisores fueron capacitados para completarlo correctamente. Se les explicaron los tipos de preguntas incluidas y cómo debía ser contestada cada sección. El comité de ética de la Universidad Autónoma de Ciudad Juárez aprobó el estudio antes de la recolección de los datos. Los participantes fueron informados sobre los objetivos de la investigación, haciendo énfasis en la confidencialidad de la información obtenida de ellos.

\subsection{Análisis de datos}

La confiabilidad de los datos fue analizada utilizando el índice Alfa de Cronbach (Nunally, 1978; Levy \& Varela, 2003) el cual nos permite conocer la consistencia interna de los datos, en este caso de los factores analizados. Debido a que los datos que se obtuvieron con el cuestionario aplicado son del tipo ordinal, se empleó la prueba estadística no (continúa) 
(continuación)

paramétrica Rangos de Wilcoxon para comparar los niveles de fatiga entre los factores analizados. De igual manera, se empleó la prueba no paramétrica Tau B de Kendall para determinar las correlaciones entre los factores y los ítems analizados. Todos los análisis de datos fueron realizados utilizando el software de estadística SPSS ${ }^{\circ}$ versión 17.

\section{RESULTADOS}

Se encuestó a un total de 121 trabajadores (42\% del total de operadores de tornos $\mathrm{CNC}$ ) que realizan actividades de carga, descarga y programación de máquinas (véase la figura 1). El promedio de edad de los trabajadores fue de 28,01 años $( \pm 6,45)$, la antigüedad en la empresa fue de 4,6 años $( \pm 5,09)$ y la antigüedad como operador de torno CNC fue de 2,6 años $( \pm 2,07)$. El cuadro 2 muestra más información demográfica de la muestra de trabajadores encuestados.

Cuadro 2

Información demográfica

\begin{tabular}{|ccc|ccc|ccc|ccc|}
\hline $\begin{array}{c}\text { Edad } \\
\text { (años) }\end{array}$ & $\mathrm{n}$ & $\%$ & $\begin{array}{c}\text { Años en la } \\
\text { empresa }\end{array}$ & $\mathrm{n}$ & $\%$ & $\begin{array}{c}\text { Años en el } \\
\text { cargo }\end{array}$ & $\mathrm{n}$ & $\%$ & Cargo & $\mathrm{n}$ & $\%$ \\
\hline$<20$ & 2 & 2 & $<1$ & 13 & 11 & $<1$ & 24 & 24 & Operador & 116 & 95 \\
$21-30$ & 77 & 71 & $1-3$ & 32 & 28 & $1-3$ & 30 & 30 & & \\
$31-40$ & 25 & 23 & $3-6$ & 53 & 46 & $3-6$ & 40 & 40 & Líder de & 6 & 5 \\
$41-50$ & 4 & 4 & $6-9$ & 3 & 3 & $6-9$ & 1 & 1 & grupo y & \\
$>51$ & 1 & 1 & $>20$ & 15 & 13 & $>20$ & 4 & 4 & operador & \\
\hline Horas & & & & & & & & & & \\
que & $\mathrm{n}$ & $\%$ & Educación & $\mathrm{n}$ & $\%$ & Estado & $\mathrm{n}$ & $\%$ & & \\
duerme & & & & & & civil & & & \\
\hline$<5$ & 3 & 3 & Primaria & 0 & 0 & Soltero & 29 & 27 & & \\
$5-6$ & 37 & 33 & Secundaria & 9 & 7,6 & Casado & 78 & 73 & & \\
$6-7$ & 39 & 35 & Preparatoria & 43 & 36,7 & Divorciado & 0 & 0 & & \\
$7-8$ & 24 & 22 & Técnico & 65 & 55,5 & & & & & \\
$>8$ & 8 & 7 & & & & & & & & \\
\hline
\end{tabular}

Elaboración propia.

Figura 1 


\section{Operadores de tornos CNC}

\subsection{Factores e ítems de fatiga}

El cuadro 3 muestra los niveles de fatiga obtenidos por los factores e ítems del cuestionario SOFI-S. También se muestra el valor del índice Alfa de Cronbach que fue calculado para los factores de fatiga, donde en 4 de los 5 factores obtuvo valores de 0.742 a 0.827 , que indican que las mediciones de fatiga tienen buena o muy buena consistencia interna (Levy et al., 2003).

\section{Cuadro 3}

Valores promedio y desviación estándar (DE) de los factores e ítems de fatiga

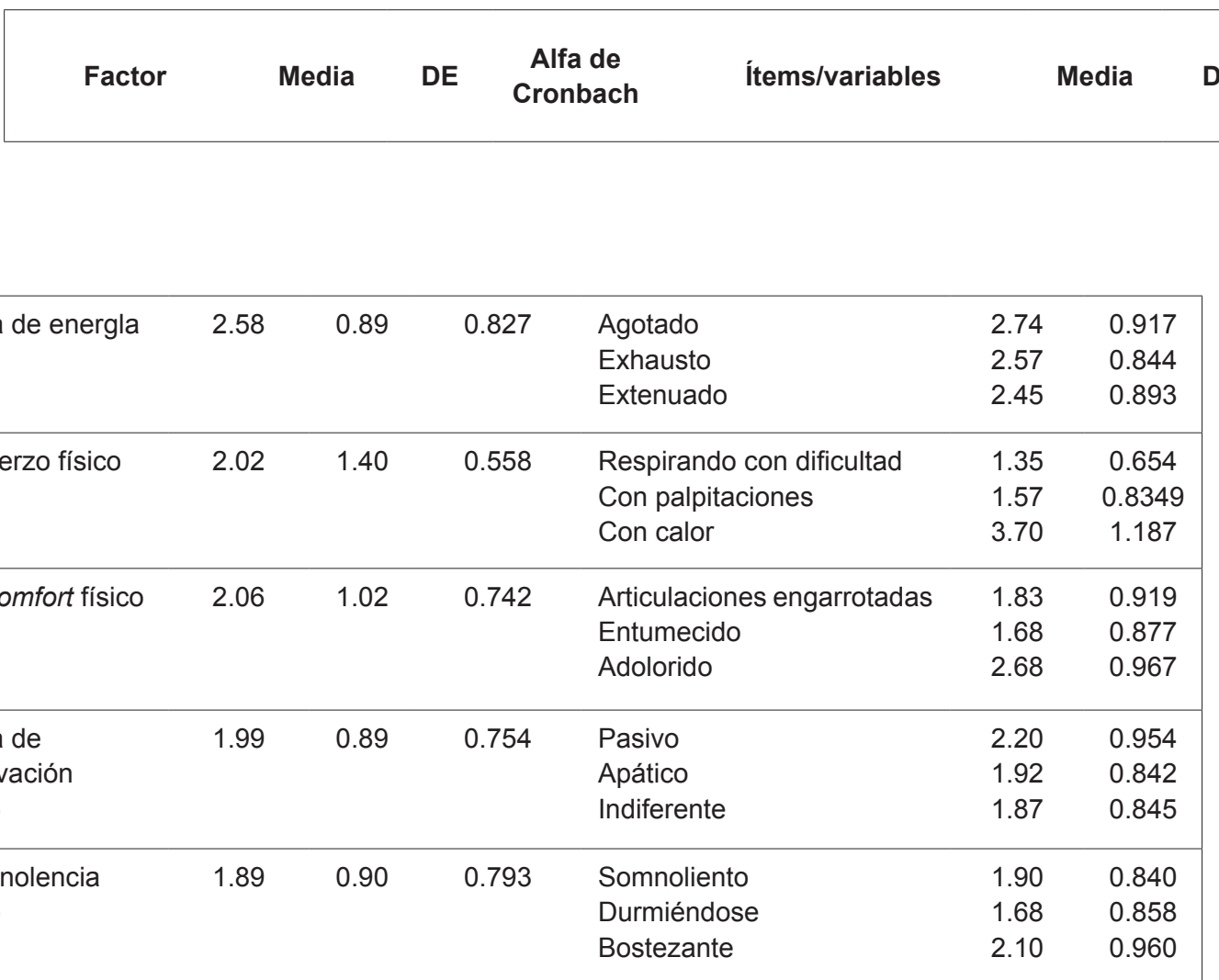

\footnotetext{
Elaboración propia.
} 
3.2 Comparación de niveles de fatiga
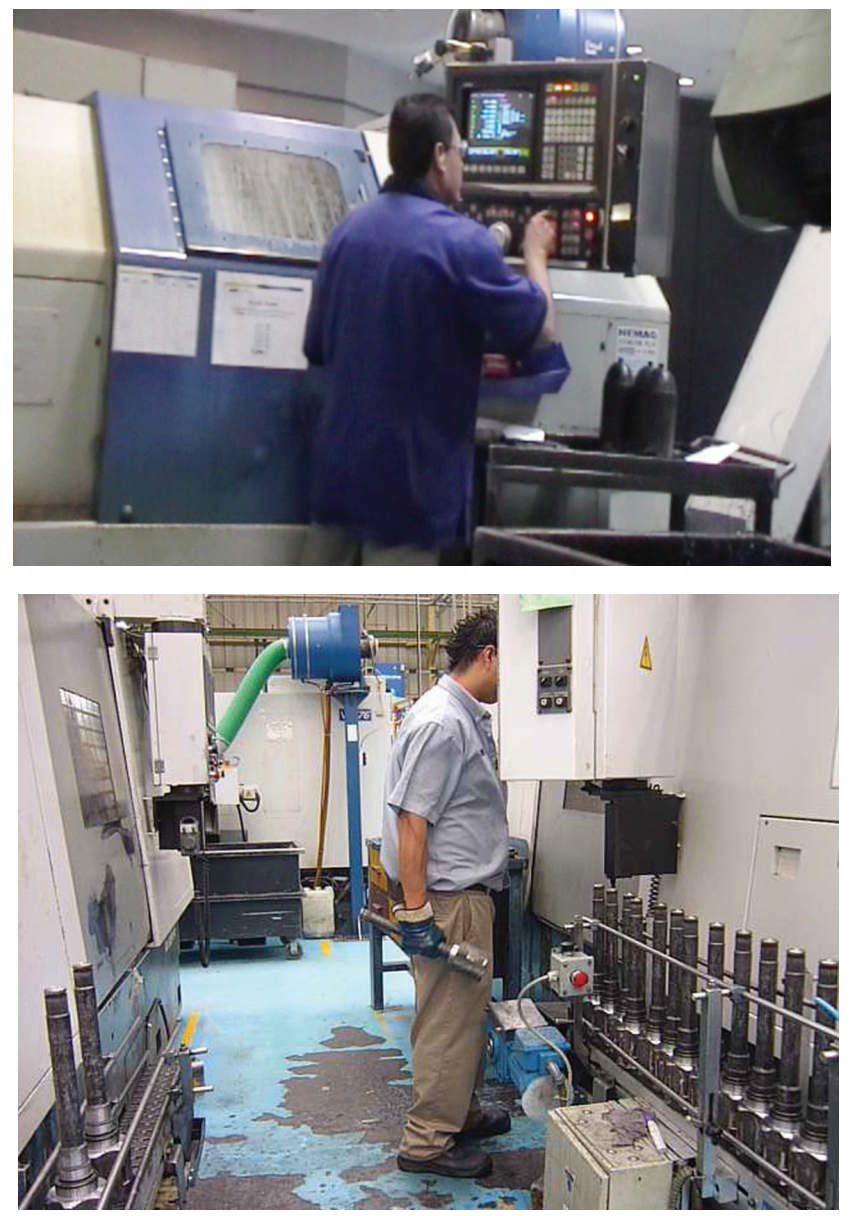

El cuadro 4 muestra el valor $p$ obtenido con la prueba Rangos de Wilcoxon de las comparaciones entre los factores de fatiga. Se puede observar que solamente las comparaciones de los factores discomfort físico vs falta de motivación y falta de motivación vs somnolencia obtuvieron valores de $p$ superiores a 0.05 , por lo tanto son las únicas en las que los niveles de fatiga se encontraron diferentes, siendo significativamente mayor el factor falta de energía a los 4 restantes. 


\section{Cuadro 4}

Comparaciones entre los factores analizados (se muestra valor pcon $\alpha=0.05$ )

\begin{tabular}{|l|c|c|c|c|}
\hline Factor & $\begin{array}{c}\text { Esfuerzo } \\
\text { físico }\end{array}$ & $\begin{array}{c}\text { Discomfort } \\
\text { físico }\end{array}$ & $\begin{array}{c}\text { Falta de } \\
\text { motivación }\end{array}$ & Somnolencia \\
\hline Falta de energía & 0.00 & 0.00 & 0.00 & 0.00 \\
Esfuerzo físico & & 0.031 & 0.001 & 0.00 \\
Discomfort físico & & & 0.235 & 0.04 \\
Falta de motivación & & & & 0.21 \\
\hline
\end{tabular}

Elaboración propia.

\subsection{Análisis de correlación}

El cuadro 5 muestra el análisis de correlaciones no paramétricas entre los factores de fatiga incluidos en el estudio. Se puede observar que todas las correlaciones resultaron significativas a un nivel de significancia de $\alpha=0.01$, indicando que las relaciones son lo suficientemente importantes para ser consideradas en el estudio (Levy et al., 2003). Siendo la correlación con el mayor valor la encontrada entre los factores falta de energía y esfuerzo físico.

\section{Cuadro 5}

Matriz de correlaciones entre factores de fatiga

\begin{tabular}{|lccccc|}
\hline Dimensión & $\begin{array}{c}\text { Falta de } \\
\text { energía }\end{array}$ & $\begin{array}{c}\text { Esfuerzo } \\
\text { físico }\end{array}$ & $\begin{array}{c}\text { Discomfort } \\
\text { físico }\end{array}$ & $\begin{array}{c}\text { Falta de } \\
\text { motivación }\end{array}$ & Somnolencia \\
\hline Falta de energía & 1.000 & $.479^{* *}$ & $.455^{\star *}$ & $.333^{* *}$ & $.328^{* *}$ \\
Esfuerzo físico & & 1.000 & $.466^{* *}$ & $.363^{* *}$ & $.410^{* *}$ \\
Discomfort físico & & & 1.000 & $.325^{* *}$ & $.391^{* *}$ \\
Falta de motivación & & & & 1.000 & $.317^{* *}$ \\
Somnolencia & & & & & 1.000 \\
\hline
\end{tabular}


** Correlación significativa con $\alpha=0.01$ (bilateral).

Elaboración propia.

El cuadro 6 muestra el análisis de correlaciones no paramétricas entre los factores e ítems de fatiga incluidas en el estudio. Se puede observar que los índices de correlación superiores a 0.7, el cual es considerado como correlación alta (Levy et al., 2003), fueron los obtenidos entre los ítems y el factor al que pertenecen, esto de acuerdo a la agrupación factorial obtenida por Ahsberg et al. (1997) y González et al. (2005). Es decir, podemos encontrar niveles de asociación más altos entre los ítems y el factor al que corresponden.

\section{Cuadro 6}

\section{Matriz de correlación entre variables y dimensiones de fatiga}

\begin{tabular}{|c|c|c|c|c|c|}
\hline \multirow{2}{*}{ Ítems } & \multicolumn{5}{|c|}{ Dimensiones de fatiga } \\
\hline & $\begin{array}{l}\text { Falta de } \\
\text { energía }\end{array}$ & $\begin{array}{l}\text { Esfuerzo } \\
\text { físico }\end{array}$ & $\begin{array}{l}\text { Discomfort } \\
\text { físico }\end{array}$ & $\begin{array}{l}\text { Falta de } \\
\text { motivación }\end{array}$ & Somnolencia \\
\hline Agotado & $.756^{* *}$ & $.468^{\star *}$ & $.463^{* *}$ & $.311^{* *}$ & $.324^{* *}$ \\
\hline Exhausto & $.793^{* *}$ & $.403^{\star *}$ & $.394^{\star *}$ & $.306^{* *}$ & $.245^{\star *}$ \\
\hline Extenuado & $.762^{\star *}$ & $.417^{* *}$ & $.380^{* *}$ & $.301^{* *}$ & $.329^{* *}$ \\
\hline Resp. difi & $.314^{* *}$ & $.541^{* *}$ & $.237^{\star *}$ & $.252^{* \star}$ & $.237^{* *}$ \\
\hline Palpi & $.356^{* *}$ & $.669^{* *}$ & $.392^{* *}$ & $.326^{* *}$ & $.379^{* *}$ \\
\hline Calor & $.396^{\star *}$ & $.685^{\star *}$ & $.373^{* *}$ & $.256^{* *}$ & $.309^{* *}$ \\
\hline Art. engarro & $.332^{* *}$ & $.348^{* *}$ & $.748^{* *}$ & $.254^{* *}$ & $.266^{* *}$ \\
\hline Entumecido & $.427^{* *}$ & $.373^{\star *}$ & $.720^{* *}$ & $.325^{* *}$ & $.295^{* *}$ \\
\hline Adolorido & $.401^{* *}$ & $.422^{* *}$ & $.696^{* *}$ & $.261^{* *}$ & $.357^{* *}$ \\
\hline Pasivo & $.245^{\star *}$ & $.212^{\star *}$ & $.247^{* *}$ & $.679^{* *}$ & $.223^{* *}$ \\
\hline Apático & $.360^{* *}$ & $.388^{* *}$ & $.348^{* *}$ & $.790^{* *}$ & $.262^{* *}$ \\
\hline Indiferente & $.312^{* *}$ & $.386^{* *}$ & $.303^{* *}$ & $.743^{\star *}$ & $.364^{* *}$ \\
\hline Somnoliento & $.358^{* *}$ & $.400^{\star *}$ & $.432^{* *}$ & $.328^{* *}$ & $.734^{* *}$ \\
\hline
\end{tabular}


Hernández, Brunette, Ibarra, García

\begin{tabular}{llllll} 
Durmiéndose & $.314^{* *}$ & $.381^{* *}$ & $.309^{\star *}$ & $.270^{\star *}$ & $.686^{* *}$ \\
Bostezante & $.173^{\star}$ & $.288^{\star *}$ & $.265^{\star *}$ & $.201^{* *}$ & $.759^{\star *}$ \\
\hline
\end{tabular}

** Correlación significativa con $\alpha=0.01$ (bilateral).

* Correlación significativa con $\mathrm{a}=0.05$ (bilateral).

Elaboración propia. 


\section{DISCUSIÓN}

Se encuestó un total de 121 operadores de tornos CNC, $42 \%$ del total de operadores que laboran en una empresa fabricante de flechas homocinéticas ubicada en Celaya, Guanajuato, en el centro de México. El 71\% del total de trabajadores encuestados tiene entre 21 y 30 años de edad, mientras que el promedio de edad es de 28 años, estos datos indican que gran parte de los trabajadores encuestados pueden considerarse como jóvenes.

Respecto de la antigüedad en la empresa y en el cargo los datos son muy similares. El 74\% de los trabajadores tiene entre 1 y 6 años laborando en la empresa y el $70 \%$ tiene entre y 1 y 6 años de experiencia como operador de tornos CNC. Esto nos indica que los trabajadores encuestados cumplieron con el criterio de tener al menos 6 meses de experiencia en el cargo actual. Un 35\% mencionó dormir menos de 7 horas, mientras que el 36\% mencionó dormir menos de 6 horas. Esta situación puede ser ocasionada por el esquema de turnos extendidos (jornadas de 12 horas durante cuatro días con 2 días de descanso) en el que se encuentra laborando la empresa. Debido al tipo de trabajo que realizan los operadores de tornos $\mathrm{CNC}$, el grado de educación que solicita la empresa es el mínimo del nivel de secundaria para estas tareas, encontrando que solamente el $7,6 \%$ ha estudiado educación primaria, mientras que el $92,2 \%$ tiene estudios de preparatoria o carrera técnica en máquinas y herramientas.

Los valores obtenidos en el índice Alfa de Cronbach fueron superiores a 0.7, que de acuerdo con Nunally (1978) y Levy et al. (2003) los datos tienen un nivel de confiabilidad muy bueno. La única excepción fue el factor esfuerzo físico, que obtuvo un índice de 0.558, el cual es considerado como poco confiable. Sin embargo, este resultado coincide con los obtenidos por Ahsberg (1997) y González (2005) y es atribuido a la variable "con calor", la cual obtuvo el valor de fatiga y la desviación estándar más altas, de 3.7 y 1.187, respectivamente. Un factor que ocasionó esta situación es que dentro de las líneas de producción, además de contar con tornos CNC, también existen hornos de tratamiento térmico que elevan significativamente la temperatura del ambiente, lo que se refleja en los resultados. A pesar de esto, los valores del índice Alfa de Cronbach, al ser consistentes con investigaciones anteriores, confirman la validez del cuestionario SOFI-S y la confiabilidad para ser aplicado para la valoración de fatiga en operadores de tornos CNC. 
El nivel más alto de fatiga fue obtenido por el factor falta de energía, con 2.58 puntos de un máximo de 5. De igual forma, el factor falta de energía fue el más alto para Ahsberg (1997) en las actividades realizadas por bomberos, maestros de grupos numerosos, estudiantes en épocas de exámenes, personal médico y operadores de sistemas de emergencias (trabajos considerados como demandantes), y para González (2005) y Barker (2011) en el trabajo de enfermeras. Sin embargo, el nivel de fatiga apenas sobrepasa el $50 \%$ de la escala utilizada, lo cual puede deberse a la edad de los trabajadores encuestados, que en promedio fue de 28 años.

En contraste, el nivel más bajo de fatiga obtenido para el trabajo realizado por los operadores de tornos $\mathrm{CNC}$ fue el registrado por el factor somnolencia, con 1.89 puntos. Situación que, de acuerdo con Ahsberg (1997), se presenta en actividades desarrolladas por bomberos y en pruebas físicas de esfuerzo máximo, pero no así en enfermeras donde el menor nivel de fatiga es el obtenido por el factor falta de motivación (González, 2005; Barker, 2011). Algunas actividades donde el factor somnolencia puede mostrar el nivel más alto de fatiga son la operación de cuartos de control en plantas nucleares y conductores de autobús (Ahsberg, 1997).

Las comparaciones de los niveles de fatiga entre los factores falta de energía y esfuerzo físico contra los tres factores restantes mostraron diferencias significativas $(\alpha=0.05)$, donde el valor del primer factor es mayor que los factores esfuerzo físico, discomfort físico, falta de motivación y somnolencia. Los factores que no mostraron diferencias fueron el discomfort físico respecto del factor falta de motivación y el factor somnolencia respecto del factor falta de motivación, estos 4 los de menor nivel de fatiga. Estos resultados coinciden con los obtenidos por Barker (2011), donde el factor falta de energía fue significativamente mayor que los 4 restantes para las actividades realizadas por enfermeras.

El análisis de correlaciones entre los 5 factores analizados mostró índices significativos $(\alpha=0.01)$ con valores entre 0.317 y 0.479 , sin embargo estos son considerados como índices de correlación baja (Levy \& Varela, 2003). En el caso del análisis de correlación entre los ítems y los factores de fatiga, se obtuvieron índices de correlación considerados como altos o muy altos (Levy \& Varela, 2003) entre los ítems y el factor al que corresponden, situación similar a la obtenida por Ahsberg (1997) y González (2005). 


\section{CONCLUSIONES}

El hecho de que se presente el mayor nivel de fatiga en el factor falta de energía indica que los trabajadores perciben disminución de fuer$z a$ (Ahsberg, 1997) como consecuencia de sus actividades, en este caso la realización de esfuerzos físicos y mentales de forma combinada al operar los tornos CNC, además de trabajar en el esquema de turnos extendidos.

La comparación estadística y los índices de correlación obtenidos entre los factores falta de energía, esfuerzo físico demuestran que la fatiga de los trabajadores se manifiesta principalmente con los síntomas de estar exhausto, agotado, extenuado y con calor, ítems que son los de mayor impacto en la fatiga de los trabajadores (Ahsberg, 1997; González et al., 2005).

Este estudio confirma la aplicabilidad del cuestionario SOFI-S en trabajadores mexicanos, contribuye a la identificación de los factores de fatiga en operadores de máquinas consideradas como TMA en países en vías de desarrollo y da pautas para nuevas investigaciones que indaguen sobre la contribución a la fatiga cuando se realizan esfuerzos físicos y mentales de forma combinada.

Se recomienda aplicar la versión en castellano del cuestionario SOFI-S en otras actividades laborales y realizar analisis factoriales y estructurales para confirmar el ajuste de los ítems y los factores incluidos.

\section{REFERENCIAS}

1. Ahsberg, E. (2000). Dimensions of fatigue in different working populations. Scandinavian Journal of Psychology, 41, 231-241.

2. Ahsberg, E., \& Fürst, C. (2001). Dimension of fatigue during radiotherapy. An aplication of the Sewdich Occupational Fatigue Inventory (SOFI) on cancer patients. Acta Oncologica, 40(1), 3743.

3. Ahsberg, E., Gamberale, F., \& Kjellberg, A. (1997). Perceived quality of fatigue during different occupational task. Development of a questionnaire. International Journal of Industrial Ergonomics, 20, 121-135. 
4. Barker, M., L., \& Nussbaum, M. (2011). Fatigue, performance and the work environment: a survey of registered nurses. Journal of Advanced Nursing, 67(6), 1370-1382.

5. Barker, L. M., \& Nusbaum, M. (2011). The effects of fatigue on performance in simulated nursing work. Ergonomics, 54(9), 815829.

6. Barrientos-Gutiérrez, T., Martínez-Alcántara, S., \& MéndezRamírez, I. (2004). Validez de constructo, confiabilidad y punto de corte de la Prueba de Síntomas de Subjetivos de Fatiga en Trabajadores Mexicanos. Salud Pública de México, 46(6), 516523.

7. Borg, G. (1990). Psychophysical scaling with applications in physical work and the perception of exertion. Scandinavian Journal of Work, Environmental and Health, 16, 55-58.

8. DiDomenico, A., \& Nussbaum, M. (2011). Effects of different physical workload parameters on mental workload and performance. International Journal of Industrial Ergonomics, 41, 255-260.

9. DiDomenico, A., \& Nussbaum, M. (2008). Interactive effects of physical and mental workload on subjective workload assessment. International Journal of Industrial Ergonomics, 38, 977-983.

10. Farrer Velázquez, F., Minaya Lozano, G., Niño Escalante, J., \& Ruiz Ripollés, M. (1994). Manual de ergonomía. Madrid: Fundación Mapfre.

11. Fürst, C., \& Ahsberg, E. (2001). Dimensions of fatigue during radiotherapy. An appication of the multidimensional fatigue inventory. Supportive Care in Cancer, 9(5), 355-360.

12. González, J. L., Moreno, J. B., Garrosa, H. A., \& López, L. A. (2005). Spanish version of the Swedish Occupational Fatigue Inventory (SOFI): factorial replication and validity. Introducción Journal of Industrial Ergonomics, 35, 737-746.

13. Hernández Arellano, J., Ibarra Mejía, G., Serratos Pérez, J. N., García Alcaraz, J. L., \& Brunette, M. J. (2012). Construction of a survey to assess workload and fatigue among AMT operators in Mexico. WORK, 41, 1790-1796. 
14. Hernández, V. M., Hernández, J. L., Serratos, J. N., \& García, J. L. (Octubre del 2009). Fatigue and work conditions in tomato packers in Jalisco State Mexico: Part II. Proceedings of the 14th Annual International Conference on Industrial Engineering, 315-320.

15. Juárez-García, A. (2007). La dimensión de fatiga-energía como indicador de presentismo: Validez de una escala en trabajadores mexicanos. Ciencia y Trabajo, 9(24), 55-60.

16. Leung, A., Chan, C., \& He, J. (2004). Structural stability and reliability of the Swedish Occupational Fatigue Inventory among Chinese VDT workers. Applied Ergonomics, 35, 233-241.

17. Michielsen, H., De Vries, J., \& Van H., G. (2003). Psychometrics qualities of a brief self-rared fatigue measure. The fatigue assessment scale. Journal of Psychosomatic Research, 54, 345-352.

18. Mital, A. (1995). The role of ergonomics in design for manufacturability and humans on general in advanced manufacturing technology: preparing the American force for global competition beyond the year 2000. International Journal of Industrial Ergonomics, $15,119-135$.

19. Mital, A., \& Pennathur, A. (2004). Advanced technologies and humans in manufacturing. International Journal of Industrial Ergonomics, 33, 295-313.

20. Mondelo, P. G. E. (1996). La ergonomía en la ingeniería de sistemas. Madrid: Isdefe.

21. Nunally, J. (1978). Psychometry theory. Nueva York: McGraw-Hill.

22. Roscoe, J., Kaufman, M., Matteson-Rusby, S., Palesh, O., Ryan, J., Kohli, S., et al. (2007). Cancer-related fatigue and sleep disorders. The Oncologist, 12 (Supplement), 35-42.

23. Seraves, P., Verhagen, C., \& Bleijenberg, G. (2002). Fatigue in cancer patients during and after treatment: Prevalence, correlates and interventions. European Journal of Cancer, 38(1), 27-43.

24. SPSS Inc., and IBM Company Headquarters, Wiewille, W. W., \& Casali, J. G. (1983). A validated rate scale for global mental 
workload measurement in a test and evaluation environment. Proceedings of the Human Factors Society 27th Annual Meeting, 129-133.

25. Winwood, P., \& Casalli, J. (2005). Developmentand validation of a scale to measure work-related fatigue and recuvery: the occupational fatigue exhaustion recovery scale (OFER). Journal of Occupational and Environmental Medicine, 47, 594-606.

26. Yoshitake, H. (1978). Three characteristics patterns of subjective fatigue symptoms. Ergonomics, 21, 231-233. 